\title{
SOME COMPLETE CYCLES ON THE $n$-CUBE
}

\author{
W. H. MILLS
}

1. Let $Q_{n}{ }^{*}$ be the $n$-dimensional hypercube (or $n$-cube) whose vertices are the $2^{n}$ vectors of dimension $n$ with components 0 's and 1 's. Let $Q_{n}$ be the graph that consists of the edges and vertices of $Q_{n}^{*}$. A complete cycle (or Hamilton circuit) on $Q_{n}$ is a cyclic path on the graph $Q_{n}$ that passes through each vertex once and only once. Let $C$ be such a cycle and let $P_{1}, P_{2}, \cdots, P_{2^{n}}=P_{0}$ be the consecutive vertices of $C$. Any two consecutive vertices $P_{i}, P_{i+1}$ differ in exactly one component. Let $A_{i}$ denote the index of that component. Then $A_{i}$ is called the $i$ th change number of $C$. The subscripts $i$ in the $P_{i}$ and the $A_{i}$ are taken modulo $2^{n}$. Clearly $C$ is determined by $P_{1}$ and the sequence of $2^{n}$ change numbers

$$
A_{1}, A_{2}, \cdots, A_{2^{n}}=A_{0} .
$$

Gilbert [1] has given a necessary and sufficient condition on (1) in order that it be the sequence of change numbers of a complete cycle on $Q_{n}$.

We will say that $C$ traverses an $r$-cube $R$ if the $2^{r}$ vertices of $R$ occur as consecutive vertices of $C$.

The complete cycles on $Q_{3}$ are easy to determine. They are all equivalent under the group of symmetries of $Q_{3}$, and each one of them traverses four squares. For $n=4$ the complete cycles have been enumerated [1]. They are of 9 distinct types, all of which traverse squares. However, one of them does not traverse any 3-cubes. For $n \geqq 5$ I will show the existence of complete cycles that do not traverse any $r$-cubes, $2 \leqq r \leqq n-1$. The proof is by induction.

2. The case $n=5$. In this case we assert that

$$
\begin{aligned}
& 1,2,3,5,2,3,4,5,3,4,1,5,4,1,2,5, \\
& 1,2,3,5,2,3,4,5,3,4,1,5,4,1,2,5
\end{aligned}
$$

is the sequence of change numbers of a complete cycle on $Q_{5}$ that does not traverse any 2,3 , or 4 -cubes. Let $A_{i}$ denote the $i$ th number in the sequence (2). We first show that this sequence is the sequence of change numbers of a complete cycle on $Q_{5}$. We may take $P_{1}=(00000)$. Then $P_{2}=(10000), \quad P_{3}=(11000), \quad P_{4}=(11100), \quad P_{5}=(11101), \quad P_{6}$ $=(10101), \cdots$ In Table I the fact that $P_{i}=(f g h j k)$ is indicated by an

Received by the editors May 31, 1962. 
$i$ in the $(f g)$ row and the $(h j k)$ column. Inspecting Table I we see that we have indeed a complete cycle.

TABLE I

A COMPLETE CyCle ON THE 5-CUBE

\begin{tabular}{rrrrrrrrr}
\hline \hline & $(000)$ & $(001)$ & $(010)$ & $(011)$ & $(100)$ & $(101)$ & $(110)$ & $(111)$ \\
\hline$(00)$ & 1 & 32 & 20 & 21 & 12 & 13 & 19 & 14 \\
$(01)$ & 26 & 31 & 27 & 22 & 25 & 24 & 18 & 23 \\
$(10)$ & 2 & 7 & 9 & 8 & 11 & 6 & 10 & 15 \\
$(11)$ & 3 & 30 & 28 & 29 & 4 & 5 & 17 & 16 \\
\hline
\end{tabular}

Now $P_{j}, P_{j+1}, P_{j+2}, P_{j+3}$ are the vertices of a square if and only if $A_{j}=A_{j+2}$. (Here the subscripts are taken modulo 32 .) A brief inspection of (2) shows that $A_{j} \neq A_{j+2}$ for all $j$. Hence our complete cycle traverses no squares.

In the sequence (2) the longest gap between successive identical integers is 10 . Hence any set of 10 consecutive integers in (2) contains all five integers $1,2,3,4,5$. The same is clearly true for any set of 15 consecutive integers in (2). This implies that our complete cycle traverses no 4 -cubes.

To complete the discussion we need to show that our complete cycle traverses no 3 -cubes.

Lemma. It is impossible to traverse a 3-cube without traversing any squares.

Proof. Suppose $P_{j}, P_{j+1}, \cdots, P_{j+6}$ all lie on a 3 -cube. Let $A_{j}, \cdots, A_{j+5}$ be the corresponding change numbers. No four of these change numbers can be distinct. Suppose no squares are traversed. Then $A_{j}, A_{j+1}, A_{j+2}$ are distinct and $A_{j+3} \neq A_{j+1}, A_{j+2}$. Hence $A_{j+3}$ $=A_{j}$. Similarly $A_{j+4}=A_{j+1}, A_{j+5}=A_{j+2}$. It follows that $P_{j+6}=P_{j}$, a contradiction. This proves the lemma.

This lemma completes the proof of the fact that the complete cycle with change numbers (2) does not traverse any 2, 3, or 4-cubes.

3. The induction from $n$ to $n+1$. Let $n \geqq 5$, and let $C_{n}$ be a complete cycle on the $n$-cube $Q_{n}$. Let $P_{1}=(000 \cdots 0), P_{2}, \cdots, P_{2^{n}}=P_{0}$ be the sequence of vertices of $C_{n}$, and let $A_{1}, A_{2}, \cdots, A_{2^{n}}=A_{0}$ be the corresponding sequence of change numbers. We suppose

(i) $C_{n}$ traverses no $r$-cubes, $2 \leqq r \leqq n-1$.

(ii) $P_{1} P_{2} P_{11} P_{12}$ are the consecutive vertices of a square $S$.

(iii) $A_{0}=4$ or $5 ; A_{2} \neq 4,5 ; A_{10}=4 ; A_{12}=5$.

We note that these conditions are satisfied by the complete cycle 
$C_{5}$ on $Q_{5}$ constructed in $\S 2$.

Set $A=A_{1}$. Then by (ii) we have $A=A_{1}=A_{11}$. Moreover, $P_{2} P_{11}$ is an edge of $Q_{n}$. Hence $P_{2}$ and $P_{11}$ differ in only one component. Let $d$ denote the index of that component. Thus the sequence of change numbers of the square $S$ is $A, d, A, d$. Moreover, since $P_{10}, P_{13}$ are not on $S$ we have $A \neq A_{10}, A_{12}$. In particular $A \neq 4,5$ by (iii). Similarly, $d \neq 4,5$. Let $\sigma$ be any fixed permutation of $1,2, \cdots, n$ such that

$$
\sigma A=d, \quad \sigma d=A, \quad \sigma 4=5, \quad \sigma 5=4 .
$$

For any vertex $P=\left(\epsilon_{1}, \epsilon_{2}, \cdots, \epsilon_{n}\right)$ of $Q_{n}$ put $\tau=\sigma^{-1}$

$$
\begin{aligned}
& \sigma P=\left(\epsilon_{\tau 1}, \epsilon_{\tau 2}, \cdots, \epsilon_{\tau n}\right), \\
& \lambda P=\left(\epsilon_{1}, \epsilon_{2}, \cdots, \epsilon_{n}, 0\right), \\
& \mu P=\left(\epsilon_{1}, \epsilon_{2}, \cdots, \epsilon_{n}, 1\right) .
\end{aligned}
$$

In particular, $\sigma P_{1}=P_{1}, \sigma P_{2}=P_{12}, \sigma P_{11}=P_{11}$, and $\sigma P_{12}=P_{2}$.

We assert that

$$
\begin{aligned}
& n+1, A_{2}, A_{3}, \cdots, A_{10}, n+1, \sigma A_{10}, \sigma A_{9}, \cdots, \sigma A_{2}, \\
& n+1, A_{12}, A_{13}, \cdots, A_{2^{n}}, n+1, \sigma A_{2^{n}}, \cdots, \sigma A_{12}
\end{aligned}
$$

is the sequence of change numbers of a complete cycle on the $(n+1)$ cube and that this complete cycle satisfies conditions (i), (ii), and (iii). Let $b_{i}$ denote the $i$ th number in the sequence (4). Thus

$$
\begin{aligned}
& b_{1}=b_{11}=b_{21}=b_{2^{n}+11}=n+1, \\
& b_{i}=A_{i}, \quad 2 \leqq i \leqq 10 \\
& b_{i}=\sigma A_{22-i}, \quad 12 \leqq i \leqq 20, \\
& b_{i}=A_{i-10}, \quad 22 \leqq i \leqq 2^{n}+10, \\
& b_{i}=\sigma A_{2^{n+1}+12-i}, \quad 2^{n}+12 \leqq i \leqq 2^{n+1} .
\end{aligned}
$$

Let $B_{1}, B_{2}, \ldots$ be the sequence of vertices of a path on $Q_{n+1}$ with sequence of change numbers (4). We may choose $B_{1}$ arbitrarily. For convenience we take

$$
B_{1}=\mu P_{2}=\mu \sigma P_{12} \text {. }
$$

Since $b_{1}=n+1$ we have $B_{2}=\lambda P_{2}$. Using (6) this gives us

$$
B_{i}=\lambda P_{i}, \quad 2 \leqq i \leqq 11 .
$$

Since $B_{11}=\lambda P_{11}$ and $b_{11}=n+1$, we have $B_{12}=\mu P_{11}=\mu \sigma P_{11}$. Now $\sigma P_{i}$ and $\sigma P_{i+1}$ differ only in the $\sigma A_{i}$ th component. Hence, using (7), we obtain $B_{i}=\mu \sigma P_{23-i}, \quad 12 \leqq i \leqq 21$. Thus $B_{21}=\mu \sigma P_{2}=\mu P_{12}$ so that 
$B_{22}=\lambda P_{12}$. From (8) we get

$$
B_{i}=\lambda P_{i-10}, \quad 22 \leqq i \leqq 2^{n}+11,
$$

in particular, $B_{2^{n}+11}=\lambda P_{1}=\lambda \sigma P_{1}$, so that $B_{2^{n}+12}=\mu \sigma P_{1}=\mu \sigma P_{2^{n}+1}$. Hence $B_{i}=\mu \sigma P_{2^{n+1}+13-i}, 2^{n}+12 \leqq i \leqq 2^{n+1}+1$, by (9). In particular $B_{2^{n+1}+1}=\mu \sigma P_{12}=B_{1}$. We see from these relations that the path $B_{1}, B_{2}, \cdots$ passes through every vertex of $Q_{n+1}$. Hence we have a complete cycle. We denote this complete cycle by $C_{n+1}$.

We note that $b_{0}=b_{2^{n+1}}=\sigma A_{12}=4 ; b_{2}=A_{2} \neq 4,5 ; b_{10}=A_{10}=4$; and $b_{12}=\sigma A_{10}=5$. Thus (iii) holds for $C_{n+1}$. Moreover, $B_{1}=\mu P_{2}, B_{2}=\lambda P_{2}$, $B_{11}=\lambda P_{11}, B_{12}=\mu P_{11}$. Since $P_{2} P_{11}$ is an edge of $Q_{n}$ it follows that $B_{1}$, $B_{2}, B_{11}, B_{12}$ are the consecutive vertices of a square. Thus (ii) holds for $C_{n+1}$. To complete the discussion we must show that (i) holds, i.e., that $C_{n+1}$ traverses no $r$-cube, $2 \leqq r \leqq n$.

Suppose $T$ is an $r$-cube traversed by $C_{n+1}, 2 \leqq r \leqq n$. It follows easily from the induction hypothesis and the sequence (4) that $T$ is not contained in the $n$-cube $\lambda Q_{n}$ or the $n$-cube $\mu Q_{n}$. Hence $T \cap \lambda Q_{n}$ is an $(r-1)$-cube. Since $C_{n+1}$ traverses $T$ it follows from (10) and (11) that $C_{n}$ traverses the $(r-1)$-cube $\lambda^{-1}\left(T \cap \lambda Q_{n}\right)$. Hence $r-1=1, r=2$, and $T$ is a square. Thus for some $j ; B_{j}, B_{j+1}, B_{j+2}, B_{j+3}$ are the vertices of the square $T$. Hence $b_{j}=b_{j+2}$. By construction $b_{j}=b_{j+2}=n+1 \mathrm{is} \mathrm{im-}$ possible. Since $T$ does not lie in either $\lambda Q_{n}$ or $\mu Q_{n}$ one of the change numbers of $T$ is $n+1$. Hence $b_{j+1}=n+1$. Therefore $j=0,10,20$, or $2^{n}+10$. Now

$$
\begin{aligned}
b_{0} & =\sigma A_{12}=4 \neq A_{2}=b_{2}, \\
b_{10} & =4 \neq 5=b_{12}, \\
b_{20} & =\sigma A_{2} \neq \sigma 4=5=A_{12}=b_{22},
\end{aligned}
$$

and finally, since $A_{2^{n}}=A_{0}=4$ or 5 we have

$$
b_{2^{n}+10}=A_{2^{n}} \neq \sigma A_{2^{n}}=b_{2^{n}+12} .
$$

It follows that $b_{j} \neq b_{j+2}$, a contradiction. Therefore, $C_{n+1}$ traverses no $r$-cubes, $2 \leqq r \leqq n$. Thus we have proved:

TheOREM. For any $n \geqq 5$ there exists a complete cycle on the $n$-cube that does not traverse any $r$-cube, $2 \leqq r \leqq n-1$.

\section{RefERENCE}

1. E. N. Gilbert, Gray codes and paths on the n-cube, Bell System Tech. J. 37 (1958), 815-826.

YALE UNIVERSITY 